\title{
Albert Einstein: un cervello in blocchetti
}

\section{Luciano Sterpellone}

\begin{abstract}
Albert Einstein, a brain in Blocks
Abstract. The Author describes the pathography of Albert Einstein, who suffered of hypertension, and died at 76 years old after the rupture of an abdominal aorta aneurysm.

After shortly referring to his relativity theory, and his involvement in the manufacturing of the first atomic bomb, the Author describes the results of the neurological and autopsic examination of Einstein's brain. The results show some particularities in the left temporal lobe that may explain his exceptional genius.
\end{abstract}

Key words: Relativity, Gedankexperiment, Arterial hypertension, Aortic aneurysm, Einstein's brain

Conflict of interest: None.

Ricevuto: 21 Aprile 2013; Accettato: 29 Aprile 2013

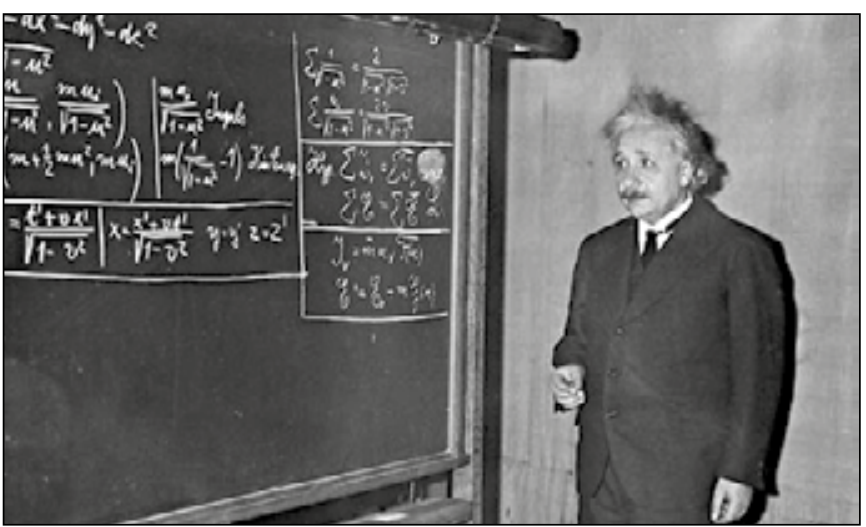

Fig. 1 -

"Due cose sono infinite: l'universo e la stupidità umana, ma riguardo all'universo ho ancora dei dubbi. La fantasia è più importante della conoscenza."

Il bambino che, secondo il pronostico paterno, "non combinerà mai nulla di buono nella vita", a ventisei anni è già famoso come un "genio" nel mondo della Scienza.

Ma Herr Hermann Einstein, modesto industriale tedesco di origine ebraica, non poteva certo prevedere una cosa del genere, dal momento che, a tre anni, il piccolo Albert non diceva ancora una parola e che fino a sette stentava a esprimersi. A dieci anni, il ragazzo trovava anche difficoltà a eseguire le più semplici operazioni aritmetiche e non figurava certo tra i primi della classe.

Unica passione il violino, con preferenza per Mozart e Vivaldi. La prima amara esperienza con il mondo la fece il giorno in cui, essendo lui l'unico ebreo nella scuola elementare, l'inse- gnante, brandendo un grosso chiodo arrugginito e mostrandolo alla scolaresca esclamò: "Ecco uno dei chiodi che gli ebrei hanno usato per crocifiggere Gesù".

Da allora il giovane Albert coverà un odioso sentimento contro l'antiebraismo (e il militarismo) tedesco, prendendosela, però, soprattutto con quegli ebrei che accettavano passivamente una situazione sempre più gravosa e che avrebbe raggiunto l'acme con l'avvento e le persecuzioni del nazismo.

Albert Einstein è nato a Ulm, nella parte sveva della Baviera, il 14 Marzo del 1879. Dopo gli studi primari e il ginnasio a Monaco, allora centro politico e intellettuale della Germania, raggiunse Milano, dove il padre conduceva una piccola azienda elettrochimica insieme al fratello Jakob, ingegnere. Ma, fallita l'impresa e non volendo rientrare in Germania, Albert si iscrisse alla facoltà di Fisica e Matematica del Politecnico di Zurigo.

Il suo primo incontro con la Scienza avvenne, in realtà, tanti anni prima, quando, a soli quattro anni, in occasione di una malattia, il padre gli regalò una bussola: il bambino rimase molto "sorpreso" dal fatto che l'ago puntava sempre in una certa direzione, il che, lì per lì, sconvolse bruscamente ogni esperienza personale.

A Zurigo, prese la laurea nel 1900, ma, senza la cittadinanza svizzera, non poteva ottenere un posto di assistente all'Università. Dovette, perciò, accontentarsi di un posticino come tecnico di terza classe all'Ufficio Brevetti di Berna, che, tuttavia, gli lasciava tempo sufficiente per dedicarsi alla ricerca. Già a quest'epoca Albert mostrava un eccezionale potere di concentrazione, riuscendo addirittura a estraniarsi del tutto nel bel mezzo di una conversazione o di una riunione per concentrarsi su di un pensiero, un'idea o un'intuizione, tanto da non accorgersi se qualcuno cercava di rivolgergli la parola. Sarebbe rimasta una delle peculiarità più spiccate della sua personalità. Ed è proprio basandosi su di essa che, nei 


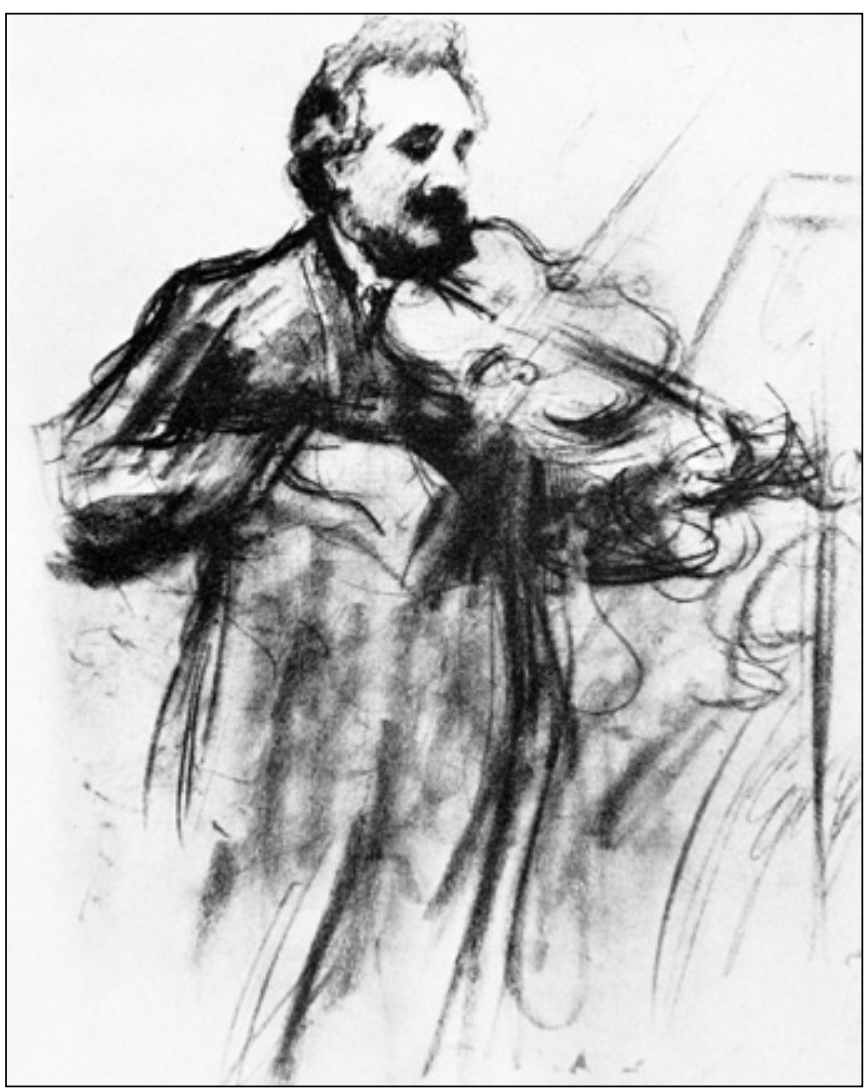

Fig. 2 - Einstein che suona il violino (disegno del russo Leonid Pasternak).

primi anni del 2000, il neuropsichiatra S. Cohen dell'Università di Cambridge decretò per lui la diagnosi di sindrome di Asperger.

Con questa, sono compatibili altre particolarità di Einstein: gli interessi ossessivi, le difficoltà nelle relazioni sociali e i problemi di comunicazione. Tutti elementi già presenti (come anche in Newton) nell'età infantile e nell'adolescenza. Einstein preferiva starsene solo e, fino a sette anni, tendeva a ripetere ossessivamente le stesse frasi. Nel mezzo di una conversazione, chiedeva venia ai presenti: "Devo pensare un po", diceva, e "perdeva lo sguardo lontano con una vaga espressione di beatitudine". A ciò si aggiunga, da adulto, la sua insofferenza per la ressa dei curiosi: a chi gli chiedeva l'autografo offriva un modulo prestampato in cui subordinava l'accoglimento della richiesta al versamento di una modesta somma in beneficenza.

Anche la tendenza di Einstein alla solitudine e la difficoltà di affrontare discorsi brevi e casuali sarebbero compatibili con la sindrome di Asperger, quantunque questa diagnosi contrasti con il suo acuto senso dell'humour ("Quanto durerà la Terra? Per ora dura da un miliardo di anni. Aspetta e vedrai...”).

\section{Tra relatività e matrimonio}

Da studente, a Zurigo, frequentava i salotti della città, esibendosi spesso con il violino (Fig. 2). A giudicare da certe foto ingiallite, doveva piacere alle ragazze: un bel giovane alto, capelli ricciuti, fossetta al mento, sguardo intenso e con buon gusto nel vestire.

Nel 1903, sposò una studentessa ungherese di matematica, incontrata al caffè Metropol, Mileva Maric, che gli avrebbe dato due figli: Eduard e Hans-Albert. Un matrimonio poco fortunato però, sia per la durezza del carattere, la scontrosità e l'eccessiva riservatezza di lei, sia, forse, perché ambedue erano troppo assorbiti dai rispettivi interessi scientifici. Né, in fondo, Albert era portato per la vita matrimoniale: "Sono un cavallo per un tiro e non uno per la pariglia o per un tiro multiplo".

Lo stesso anno del matrimonio comparve il suo primo articolo (sul fenomeno della capillarità), seguito, negli anni successivi, da altri importanti lavori sulla struttura atomico-molecolare della materia, sulla propagazione della luce e sull'elettrodinamica dei corpi in movimento, nonché, nel 1909 , dallo storico lavoro sulla teoria della relatività "speciale" o "ristretta", che confermava la sua fama nel mondo scientifico: Albert Einstein era appena trentenne.

\section{Quando la relatività è "ristretta"}

Galileo aveva fatto un esempio molto eloquente: se, nella cabina sotto-coperta di una nave (di un "navillo") ferma vi sono una vaschetta con dei pesci che nuotano, delle mosche e delle farfalle che volano e una persona che salta, i movimenti dei pesci, il volare delle mosche e delle farfalle, nonché i salti della persona saranno sempre gli stessi anche se la nave comincia a muoversi con moto uniforme. E nessuno, stando chiuso nella cabina, senza oblò, "potrebbe dire se il navillo è in movimento o se sta fermo...".

Ergo, i movimenti dei pesci e così via nella cabina sono assoluti.

Ma, per il giovane fisico, non aveva invece senso, nell'esempio dato, parlare di movimento assoluto. Si trattava, piuttosto, di un movimento relativo a qualcosa: infatti le mosche e le farfalle si muovono rispetto alla cabina della nave, presunta (la cabina) come ferma; ma la nave sta, a sua volta, muovendosi rispetto alla Terra (assunta anch'essa come ferma). E, ancora: un treno in marcia si muove rispetto a una macchina ferma al passaggio a livello, ma la macchina ferma si muove rispetto a un viaggiatore che la guarda allontanarsi mentre sta affacciato al finestrino del treno in marcia; e si allontana da lui alla stessa velocità, ma in senso opposto, con cui egli sta allontanandosi dalla macchina. In altre parole, ha senso parlare di movimento soltanto relativamente a un sistema di riferimento considerato come fermo.

Un altro esempio, di quelli che lo stesso scienziato chiama Gedankexperiment, esperimento ideale. Un viaggiatore sta mangiando nel vagone ristorante di un treno in movimento: mangia in successione dapprima la minestra, poi il secondo e, infine, la frutta. Tali eventi si verificano tutti nello stesso luogo (tavolino), ma in momenti successivi. Tuttavia, se un osservatore ideale posto lungo la strada ferrata osservasse la scena, constaterebbe che il viaggiatore consuma la minestra e la frutta a distanza di qualche chilometro l'una dall'altra. 
Crollava, pertanto, anche il concetto espresso da Newton di "tempo assoluto".

Tuttavia, tutto ciò non significa che Einstein voglia intendere che "tutto è relativo": il suo intento è, piuttosto, quello di escludere dalle leggi della fisica quanto è puramente relativo, per giungere a una sistemazione delle leggi fisiche completamente indipendente dalle condizioni dell'osservatore.

\section{Guai fisici}

Einstein era un uomo alto 1 metro e 76, con le spalle larghe, i capelli ormai quasi del tutto canuti, la testa massiccia e la muscolatura forte, ma non era affatto grasso. Portava di rado la cravatta e i calzini; fumava la pipa e non beveva quasi mai alcolici.

Apparentemente la sua salute era buona, ma, in realtà, egli soffriva di ipertensione arteriosa, che sarebbe stata la causa, nel tempo, di un'arteriosclerosi generalizzata e di una lesione aortica che lo avrebbe portato alla morte. Del resto, a quel tempo si era ben lontani da un trattamento vero e proprio dell'ipertensione arteriosa: lo sfigmomanometro era stato inventato da poco e il quadro nosologico dell'ipertensione stessa era ancora nebuloso. Negli anni '20, la terapia si basava quasi unicamente sulla dieta povera di sale e sull'impiego del tetraetilammonio e del tiocianato di potassio; i diuretici mercuriali non erano ancora entrati nell'uso comune, mentre, per gli altri diuretici, per l'idralazina e per la reserpina (per non parlare degli ACE-inibitori e degli antagonisti dei recettori AT1 dell'angiotensina II) si sarebbero dovuti attendere vari decenni.

Il che avrebbe fatto sì che l'ipertensione avrebbe continuato a minare le arterie dell'ormai quarantenne scienziato.

Durante l'anno 1928 sopravvenne "una lunga malattia" che, oggi, alcuni patografi individuano in una miocardite, malattia che lo allontanò temporaneamente dal lavoro e che lo indusse ad assumere una segretaria, la (poi) famosa Fräulein Rosa Dukas, a capo dell'Organizzazione Ebraica di Berlino.

\section{La relatività "generale"}

Nello stesso 1909, anno in cui comparve il suo lavoro sulla relatività ristretta, venne chiamato alla cattedra di Fisica teorica dell'Università di Zurigo e potè, quindi, permettersi di abbandonare il posto di impiegato all'Ufficio Brevetti di Berna. Ma, ben presto, la sua vita accademica si spostò a Praga, poi di nuovo a Zurigo e, da lì, a Berlino al Kaiser Wilhelm Institut, dove sarebbe rimasto per diciassette anni. Nel frattempo, divorziato da Mileva, sposò la cugina Elsa Löwenthal, anch'ella divorziata e madre di due ragazze.

$\mathrm{Ma}$, praticamente isolato per l'aperta opposizione alla politica del governo centrale, approfittò degli anni della I Guerra Mondiale per dare una formulazione definitiva alla nuova teoria della relatività "generale" (1919). Due anni dopo gli venne assegnato il Premio Nobel per la Fisica. Ma non, come molti credono, per la teoria della relatività, bensì per il lavoro sull" Elettrodinamica dei corpi in movimento", che aveva pubblicato sulla rivista Annalen der Physik nel 1905 , a soli 26 anni.

Anche in occasione del premio Nobel, Einstein mostrò la sua profonda semplicità d'animo e il suo disinteresse per il denaro: versò la metà del ricco Premio alla prima moglie e l'altra ai poveri di Berlino.

All'inizio del XX secolo, quando Einstein dimostrò che il limite massimo e insuperabile della velocità era quello raggiunto dalla luce, stavano comparendo nuovi innovativi mezzi di trasporto: le automobili, le navi e gli aerei promettevano ormai spostamenti sempre più rapidi. Ma, anche nell'immaginario popolare, per quanto tali, gli scienziati non sarebbero mai riusciti a costruire mezzi più veloci della luce!

Queste e altre teorie sarebbero state diffuse, spesso impropriamente, anche sulla stampa laica e non di rado sarebbero state fraintese e banalizzate. Malgrado ciò lo stesso scienziato non avrebbe perso occasione per cercare di renderle più comprensibili. Avrebbe risposto ai giornalisti che gli chiedevano di spiegarle in parole semplici: "Una volta si credeva che, se tutte le cose materiali fossero sparite, il tempo e lo spazio sarebbero rimasti. Invece, secondo la teoria della relatività, il tempo e lo spazio dovrebbero sparire insieme alle cose".

Ancora più semplice e immediata la risposta data ai ragazzi di una scuola: "La relatività? Pensate che cosa sono un minuto con i piedi sui carboni ardenti e un minuto con una bella ragazza su un prato...".

\section{Esule in America}

Negli anni della lunga permanenza a Berlino, l'ormai famoso fisico compì lunghi viaggi di studio: ritornò in Italia e a Praga, visitò l'Olanda, l'Austria, la Francia e il Medio e l'Estremo Oriente. A New York venne accolto come un eroe o una star di Hollywood, anche se, scrisse un giornale, "quando scese dalla nave, i suoi lunghi capelli bianchi e la custodia dello strumento che portava in mano lo facevano assomigliare più a un musicante che a uno scienziato".

Poi, nel 1932, pochi mesi prima dell'avvento al potere di Hitler, visto che, in Germania, l'antisemitismo si era consolidato, si trasferì dapprima in Belgio e, dopo un anno, definitivamente negli Stati Uniti, scegliendo come residenza la cittadina di Princeton nel New Jersey, sede dell'Istituto di Studi Superiori. Tutti i suoi beni rimasti in Germania sarebbero stati confiscati e i suoi scritti dati alle fiamme nella piazza antistante l'Opera di Stato.

Poco prima di emigrare disse: "Qui la mia situazione è come quella di un uomo che giace su un bel letto, dove viene torturato dalle pulci". Saputo che il partito nazista aveva messo su di lui una taglia di 50.000 marchi esclamò: "Non sapevo che la mia testa valesse tanto!".

$\mathrm{Ma}$, forse, sapeva già di avere aperto una nuova era: l'era atomica.

\section{Quando l'ipertensione non perdona}

Il 31 Dicembre del 1948 lo scienziato fu scosso improvvisamente da una serie di violenti dolori all'addome, accom- 


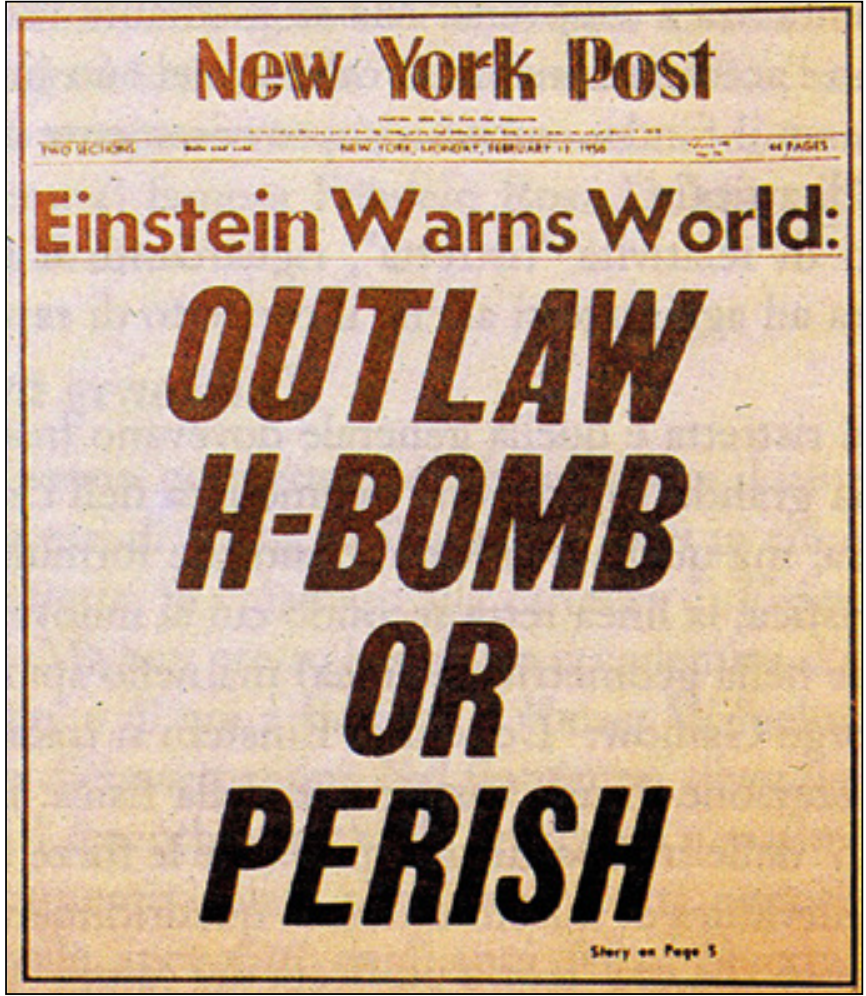

Fig. 3 -

pagnati da vomito. All'intervento chirurgico, il Professor Rudolf Nissen, allievo del famoso chirurgo Sauerbruch, scoprì che tutto era dovuto a una cirrosi epatica. Ma, nello stesso tempo, accertò a sorpresa l'esistenza di un aneurisma dell'aorta addominale. Evidentemente, l'ipertensione aveva continuato negli anni a operare in silenzio, provocando arteriosclerosi e interessando in modo particolare il segmento addominale dell'aorta, con la conseguente formazione di un aneurisma.

Quanto alla cirrosi, se si esclude la sua natura alcolica in quanto Einstein era quasi totalmente astemio, la si può agevolmente ricondurre a un'epatite itterica verosimilmente infettiva (al tempo non esisteva nemmeno il concetto di epatite virale), da lui sofferta nel 1917.

\section{"Se l'avessi saputo..."}

Pochi anni dopo essersi insediato negli Stati Uniti, Einstein si sentì completamente solo: Elsa, sconvolta dalla morte di una delle due figlie avute dal primo marito, la seguì presto nella tomba.

Quel che ora più preoccupava lo scienziato era la straordinaria scoperta compiuta da due scienziati tedeschi, Otto Hahn e Fritz Strassmann: un atomo di uranio colpito da un neutrone si spezza in due parti e libera una straordinaria quantità di energia.

In realtà, la notizia di questa scoperta non avrebbe dovuto sconvolgerlo più di tanto, anche perché era, anzi, la conferma di un corollario delle proprie teorie: ogni massa rappresenta un'energia latente e l'energia possiede una massa.

Così, mentre la fisica classica del XIX secolo aveva stabilito due leggi distinte (la conservazione della massa e la conservazione dell'energia), Einstein parlava ora di un principio unico: il principio della conservazione della massa-energia, fissando la legge di questa equivalenza nella famosa formula matematica $\mathrm{E}=\mathrm{mc}^{2}$, dove $E$ rappresenta l'energia totale, $m$ la massa e $c$ la velocità della luce. Questa formula indica, in sostanza, che ogni unità di massa rappresenta un enorme deposito di energia.

Quindi, la notizia della scoperta di Hahn e Strassmann non era tanto nella scoperta, quanto nel fatto che, di conseguenza, gli scienziati del III Reich avrebbero potuto utilizzare la fissione dell'atomo a scopo bellico utilizzando la "reazione a catena" dimostrata da E. Fermi, da L. Szilard e dall'austriaca Lisa Meitner (purtroppo dimenticata).

Il 2 Agosto del 1939, quattro settimane prima che Hitler scatenasse la II Guerra Mondiale, Einstein scrisse direttamente al presidente Roosevelt una lettera, nella quale lo avvertiva senza mezzi termini che era "possibile effettuare reazioni nucleari a catena in una forte massa di uranio, da cui verrebbero generate enormi quantità di energia e di elementi simili al radio. La conoscenza di questo nuovo fenomeno potrebbe portare alla costruzione di bombe di estrema potenza. Apprendo che la Germania ha interrotto attualmente le vendite di uranio delle miniere cecoslovacche di cui si è impossessata...".

Il Presidente rispose il 19 Ottobre. Era il via alla costruzione della bomba atomica.

Il fisico, pacifista e internazionalista per costituzione, si sarebbe pentito amaramente per sempre di aver scritto quella lettera: "Se avessi saputo che i nazisti non sarebbero riusciti a fabbricare la bomba atomica prima degli Alleati, mi sarei astenuto da qualsiasi azione" (Fig. 3).

"Se avessi saputo..." avrebbe ripetuto mille volte, sino al suo ultimo giorno.

E, dopo Hiroshima e Nagasaki, si sarebbe battuto con veemenza per convincere inglesi e americani a svelare all' $U$ nione Sovietica il segreto della bomba atomica, attirandosi l'accusa di tradimento e di filocomunismo.

\section{Sezioni di cervello}

A quanto si apprende da un appunto dello stesso Einstein scritto sei settimane prima di morire, nell'Ottobre del 1954, egli ha sofferto di un'anemia secondaria (a suo avviso "di origine virale"), "che ha decurtato del 45\% l'apporto di ossigeno" e che è stata "curata" con il cortisone... In fondo, lo scienziato non ha mai mostrato grande dimestichezza con la Medicina, né, tantomeno, fiducia in essa, forse conscio che i processi biologici non possono essere racchiusi in forme matematiche. Sosteneva, non senza convinzione: "Si può anche morire senza l'aiuto dei medici".

Il 12 Aprile del 1955 ricomparvero dolori molto violenti all'addome. Venne somministrata morfina e il paziente fu 
ricoverato per l'intervento all'Ospedale di Princeton, con sospetta rottura di aneurisma.

Poco dopo la mezzanotte del 18 Aprile, l'infermiera si accorse che egli respirava molto male; poi cominciò a parlare, ma si espresse in tedesco, per cui lei non avrebbe saputo mai riferire che cosa avesse detto. Alle ore 1:15, Albert Einstein esalò l'ultimo respiro.

La diagnosi, confermata all'autopsia, è stata "rottura di aneurisma dell'aorta addominale con emorragia massiva nello spazio retro-peritoneale".

Nel corso dell'autopsia (eseguita sette ore dopo la morte) è stato asportato il cervello per un esame dettagliato della sua struttura macroscopica e istologica. Il resto del corpo venne cremato e le ceneri furono disperse in un luogo segreto (lo stesso Einstein non era contrario a che il suo cervello venisse studiato dopo la morte e già nel 1951 aveva acconsentito di sottoporsi a particolari indagini elettroencefalografiche).

L'organo venne sezionato in 240 blocchetti di circa $10 \mathrm{~cm}^{3}$ ciascuno e conservato sotto formalina $10 \%$ in un vaso di vetro nello studio dell'anatomopatologo Thomas Harvey. Si cercava di sciogliere il quesito: il cervello di un "genio" è diverso da quello di una persona normale? Come ha fatto Einstein, "senza alzarsi dal desco" a "pesare l'universo e a dedurre che lo spazio è curvo e che il tempo può rallentare?". Certamente quel cervello doveva avere qualcosa di eccezionale...

Per giorni i media avrebbero discusso sul tema, in attesa di conoscere i risultati degli esami. Ma questi avrebbero tardato a venire. Anzi, non sarebbero arrivati per alcuni decenni...

Finalmente, nel 1985, alcuni blocchetti del cervello di Einstein vennero distribuiti a un team di neuro-anatomisti, le cui precedenti ricerche sui ratti avevano suggerito che un'aumentata attività mentale si associava a un aumento di certi tipi di cellule della glia.

Conferma: anche le indagini effettuate sulle quattro sezioni del cervello di Einstein evidenziavano un maggior numero di tali cellule.

Sarebbero, tuttavia, trascorsi altri quindici anni, finché, nell'estate del 2000, quarantaquattro anni dopo la morte dello scienziato, furono resi noti sul Lancet da parte di ricercatori della McMaster University i risultati di un accurato studio da loro condotto sul cervello dello scienziato, messi a confronto con quelli raccolti su 35 cervelli di uomini precedentemente morti alla stessa età (76 anni) e su 56 cervelli di donne coetanee, tutte persone con un'intelligenza medio-normale.

Primo reperto: i lobi parietali (in natura intimamente connessi con il ragionamento spaziale e matematico) sono risultati più estesi nel cervello di Einstein che in quello dei controlli.

Inoltre, il cervello di Einstein (risultato del 15\% più grande che nei controlli maschili, ma di peso simile) ha mostrato un certo grado di atrofia intorno alle grandi scissure nelle regioni centrali di ambedue gli emisferi, reperto questo tuttavia frequente negli individui nell'ottavo decennio di vita. Ma un reperto morfologico parti- colare, non riscontrato nei controlli, è stato che il ramo ascendente posteriore della scissura di Silvio confluisce direttamente nella scissura post-centrale, per cui manca l'opercolo parietale (la parte anteriore della circonvoluzione marginale), che normalmente si sviluppa tra le due scissure durante la vita fetale.

Ora, essendosi il maggiore sviluppo dei lobi temporali verificato in epoca molto precoce, sia longitudinalmente che in ampiezza, ciò ha limitato lo sviluppo dell'espansione posteriore della scissura di Silvio e quello dell'opercolo parietale, con conseguente sviluppo maggiore del lobulo parietale inferiore. Altra conseguenza è che tutta la circonvoluzione sopramarginale è situata dietro la scissura di Silvio, la quale risulta non divisa da un solco profondo, come accade normalmente.

Partendo dall'assunto di van Essen che una circonvoluzione si sviluppa in una regione della corteccia correlata a una funzione per consentire un'efficiente connessione assonica tra le pareti corticali opposte della circonvoluzione e che, viceversa, le scissure separano regioni corticali che hanno meno relazioni funzionali tra di loro, ne deriva che la compattezza del giro sopramarginale di Einstein nel lobulo parietale inferiore può esprimere un'area straordinariamente ampia di corteccia altamente integrata con una rete funzionale. Il che, secondo la pregressa intuizione di Ramon y Cajal, sarebbe intimamente correlato al grado di intelligenza.

In altre parole, la capacità di trasmissione degli impulsi psichici e delle associazioni tra un punto e l'altro del cervello risulta, in tal caso, più immediata, veloce ed efficace. Pertanto, secondo queste ricerche, l'eccezionale intelletto di Einstein potrebbe essere correlato a un'atipia anatomica dei suoi lobuli parietali inferiori, notoriamente connessi alle associazioni degli stimoli audio-visivi, alle sensazioni visuo-spaziali, al pensiero matematico e alla percezione dei movimenti.

I dati relativi alla cito-architettonica del suo cervello non indicano, d'altra parte, differenze nel numero dei neuroni nella compagine della corteccia nei lobi frontale e temporali, mentre potrebbe esistere una differenza nel rapporto tra cellule gliali e neuroni nella corteccia parietale sinistra. Ovviamente, tali reperti non risolvono il secolare quesito intorno a un possibile substrato neuro-anatomico dell' intelligenza. Suggeriscono, comunque, che una peculiarità di specifiche funzioni cognitive può associarsi a particolarità della struttura delle regioni cerebrali che mediano tali funzioni.

Ma, si sa, "genio" non è soltanto chi è intelligente o chi sa tante cose. Genio è soprattutto chi, sapendo certe cose, riesce a farle fruttare a favore della Scienza. "Il genio, sostiene lo stesso Einstein, è colui che migliora il sapere". Principio questo che, a suo avviso, non vale soltanto per $\mathrm{i}$ "geni", ma riveste un significato più generale: "Il valore di un uomo, per la comunità in cui vive, dipende prima di tutto dalla misura in cui i suoi sentimenti, i suoi pensieri e le sue azioni contribuiscono allo sviluppo dell'esistenza degli altri individui”. 


\section{Riassunto}

L'Autore descrive le caratteristiche di Albert Einstein nelle varie età sin da bambino, focalizzando l'attenzione sugli studi del fisico ma anche dandone un rapido profilo pato-biografico: affetto da ipertensione arteriosa, muore a 76 anni per rottura di un aneurisma dell'aorta addominale, complicanza dell'ipertensione arteriosa e della conseguente arteriosclerosi. Dopo un accenno alla teoria della relatività e al ruolo da lui svolto nella realizzazione della prima bomba atomica, l'Autore riferisce i risultati delle ricerche neuro-morfologiche effettuate a distanza di anni su sezioni autotopsic dell'encefalo dello scienziato. Queste indagini hanno messo in rilievo alcune peculiarità nell'ambito del lobo temporale sinistro, che renderebbero ragione (almeno in parte) dell'eccezionale "genialità" del grande fisico tedesco.

Parole chiave: Relatività, Gedankexperiment, Ipertensione, Aneurisma aortico, Encefalo di Einstein

Dichiarazione di conflitto di interessi: L'Autore dichiara di non avere conflitto di interessi.

Indirizzo dell'Autore:

Dr. Luciano Sterpellone

Via Nicotera 29

00195 Roma

1.sterpellone@libero.it 